\title{
Lipid Peroxidation, Caspase-3 Immunoreactivity, and Pyknosis in Late-Gestation Fetal Sheep Brain after Umbilical Cord Occlusion
}

\author{
MARGIE CASTILLO-MELÉNDEZ, JO ANN CHOW, AND DAVID W. WALKER \\ Department of Physiology, School of Biomedical Sciences, Monash University, Clayton, Victoria, 3800, \\ Australia
}

\begin{abstract}
ABSTR
Umbilical cord occlusion (UCO), a known risk factor for
perinatal brain damage, causes severe fetal asphyxia leading to
oxidative stress, lipid peroxidation, and cell death. We have
determined the effects of two 10-min UCO on the distribution of
the lipid peroxidation marker 4-hydroxynonenal (4-HNE) and
the activated form of the apoptosis marker caspase-3 in the brains
of late-gestation fetal sheep. UCO caused asphyxia, hyperten-
sion, and bradycardia, but these parameters normalized 2 h after
the occlusion. At postmortem, 48 h after the second UCO there
were significantly higher numbers of 4-HNE-positive cells in all
layers of the hippocampus and cerebellum, the parietal cortex,
substantia nigra, caudate nucleus, putamen, and thalamus com-
pared with control brains. 4-HNE immunoreactivity was also
found in white matter tracts of the subcallosal bundle, external
medullary lamina, reticular thalamic nucleus, and cerebellar fiber
tracts only in UCO brains. Double-labeling identified these cells
as predominantly neurons and astrocytes, with oligodendrocytes
showing lower levels of 4-HNE immunoreactivity. After UCO,
the number of caspase-3-immunoposotive cells was increased
significantly in the hippocampal CA1, molecular layer and den-
tate gyrus, ventrolateral thalamic nucleus, substantia nigra, pu-
\end{abstract}
tamen, and cerebellar granular and molecular layers compared with controls. Double-labeling revealed caspase- 3 immunoreactivity was mainly in neurons, and to lesser extent in astrocytes and oligodendrocytes. Pyknotic cell numbers were significantly increased in hippocampal CA1 and CA3, parietal cortex, caudate nucleus, putamen, and cerebellar Purkinje cells after UCO. These data indicate that brief asphyxia induces widespread lipid peroxidation involving all cell types of the fetal brain and apoptosis in both neurons and glia. (Pediatr Res 55: 864-871, 2004)
Abbreviations
UCO, umbilical cord occlusion
4-HNE, 4-hydroxynonenal
ROS, reactive oxygen species
TBARS, thiobarbituric acid-reactive substances
PFA, paraformaldehyde
NGS, normal goat serum
GFAP, glial fibrillary acidic protein
CNPase, 2', 3'-cyclic nucleotide 3'-phosphodiesterase
MAP-2, microtubule associated protein

Asphyxia and hypoxia are threats faced by the fetus in utero and are implicated in long-term postnatal sequelae such as mental retardation, seizure disorders and cerebral palsy (1). In particular, white matter injury is thought to result from insults that cause CNS inflammation and oxidative stress $(2,3)$. The brain is relatively deficient in oxidative defenses and highly susceptible to free radical damage (4). ROS alter the structure and function of proteins and DNA, and activate cysteine proteases (caspases), leading to apoptotic cell death (5). Hy-

Received October 9, 2002; accepted October 15, 2003.

Correspondence: M. Castillo-Meléndez, Ph.D., School of Biomedical Sciences, Monash University, Clayton, Victoria, Australia, 3800; e-mail: Margie.CastilloMelendez@med.

monash.edu.au

Supported by a grant from the National Health and Medical Research Council of Australia (D.W.W.).

DOI: 10.1203/01.PDR.0000115679.86566.C4 droxyl radical, in particular, causes lipid peroxidation by rearranging double bonds in fatty acid side chains, and other radicals trigger the destruction of adjacent fatty acid molecules (4). Peroxidation of lipids leads to formation of cytotoxic reactive aldehydes, including malondialdehyde and 4-HNE (6). Cell membrane lipid composition makes some brain regions more susceptible to damage by ROS.

Free radical generation has been shown to increase during hypoxia and reperfusion in the fetal guinea pig (7) and fetal sheep brain $(8-10)$. The fetus derives all oxygen and nutrient supply through the umbilical circulation. Partial or complete occlusions of the umbilical cord are events that threaten the fetus, as in cases of cord prolapse or cord entanglement around the fetal neck or a limb. Fetal asphyxia, hypotension, and decreased cerebral blood flow all occur as a result of obstructing umbilical blood flow (11), thus creating conditions that favor increased production of ROS and peroxidation of lipids 
in the brain. A previous study using partial but prolonged UCO in fetal sheep showed that lipid peroxides, measured as TBARS extracted from the brain, were increased in gray and white matter (12). A higher degree of lipid peroxidation was reported in white matter, attributable to the higher levels of polyunsaturated fatty acid content of these regions.

The accumulation of lipid peroxides in specific brain regions after intrauterine asphyxial stress, and its relationship to the distribution of apoptosis and cell death in the fetal brain has not been studied in detail. In the adult brain, 4-HNE is increased in neurons after oxidative stress, and both in vivo and in vitro studies suggest this lipid peroxide can induce neuronal and oligodendrocyte cell death (13-15) and therefore contribute to white matter damage. Recent reports suggest that cell death after hypoxia (16) or cytokine-mediated inflammation (17) in the neonatal brain occurs by both necrosis (sudden cell death) and apoptosis (programmed cell death). In this study, we examined the distributions of 4-HNE and caspase-3 immunoreactivities and pyknosis in the brains of fetal sheep subjected to brief $(10 \mathrm{~min})$ occlusion of the umbilical cord on two occasions.

\section{METHODS}

Animals and surgery. Eleven pregnant Border-Leicester ewes at 124-126 d gestation were used in these studies. All procedures described below had received prior approval from the Monash University Animal Ethics Committee. Anesthesia was induced in each ewe by a bolus injection of $1 \mathrm{~g}$ sodium pentothal in $20 \mathrm{~mL}$ sterile water into a jugular vein. The ewe was immediately intubated and anesthesia was maintained by inhalation of $1-2 \%$ halothane in oxygen using a closed-circuit positive pressure ventilator. Under sterile conditions, the fetal head was exteriorized through a midline abdominal and uterine incision, and polyvinyl catheters (outer diameter $1.52 \mathrm{~mm}$, inner diameter $0.81 \mathrm{~mm}$ ) filled with saline containing heparin $(50 \mathrm{IU} / \mathrm{mL})$ were inserted into a carotid artery and jugular vein. A third saline-filled catheter was placed in the amniotic sac for pressure reference. Two inflatable silastic cuffs (type OC16, In Vivo Metric, Healdsburg, CA, U.S.A.) were placed around the abdominal end of the umbilical cord. The fetus was then returned to the uterus and the incision closed. All catheters were exteriorized through a maternal flank incision, and the maternal abdomen incision was closed in layers.

Experimental procedures. After a 3-d recovery period, continuous recording of the fetal heart rate and blood pressure was commenced. Fetal arterial blood pressure and heart rate were recorded digitally using a MacLab analogue-digital converter (ADInstruments Pty. Ltd., Castle Hill, NSW, Australia) and stored and displayed on a Macintosh computer. On the day of the experiment, the umbilical cord was occluded completely for $10 \mathrm{~min}$ by inflating both cuffs with $1.5 \mathrm{~mL}$ of sterile water ( $n=6$ fetuses). Fetal arterial blood samples $(0.6 \mathrm{~mL})$ were drawn anaerobically at 30,20 , and 10 min before the occlusion, at 5 and 10 min during the occlusion, and then at intervals up to $48 \mathrm{~h}$ after the occlusion for measurement of $\mathrm{pH}, \mathrm{PO}_{2}, \mathrm{PCO}_{2}$, and $\mathrm{O}_{2}$ saturation using a Radiometer ABL 5 blood gas analyzer (Radiometer Pacific Pty. Ltd., Blackburn, Victoria, Aus- tralia). Control animals $(n=5)$ did not undergo cord occlusion but were subjected to the same sampling protocol. The experimental protocol was carried out twice in each fetus, on postoperative $\mathrm{d} 3$ and 5 . All ewes and fetuses were killed by sodium pentobarbitone (Lethabarb, Virbac Pty. Ltd., Peakhurst, NSW, Australia) overdose given to the ewe $48 \mathrm{~h}$ after the second occlusion. The fetal brain was perfused transcardially with approximately $1 \mathrm{~L}$ cold saline, and then with approximately $1 \mathrm{~L} \mathrm{4 \%} \mathrm{PFA} \mathrm{in} \mathrm{PBS} \mathrm{(pH} \mathrm{7.4).} \mathrm{The} \mathrm{brain} \mathrm{was} \mathrm{then}$ removed from the skull, postfixed in $4 \%$ PFA for $12 \mathrm{~h}$, and then transferred to $4 \%$ PFA containing $30 \%$ sucrose until processing.

Immunohistochemistry. Immunohistochemistry was carried out on $8-\mu \mathrm{m}$ paraffin-embedded sections. Sections were first deparaffinized in xylene, and antigen retrieval was carried out in $0.01 \mathrm{M}$ citric acid buffer ( $\mathrm{pH}$ 6) using a microwave oven (3 $\times 2 \mathrm{~min}$ ). The sections were then rinsed in PBS, incubated in $0.3 \%$ hydrogen peroxide for $20 \mathrm{~min}$ at room temperature to block endogenous peroxidase activity, incubated with 3\% NGS in $0.1 \mathrm{M}$ PBS containing 3\% skim milk for $45 \mathrm{~min}$ at room temperature to block nonspecific binding, and then incubated overnight at $4{ }^{\circ} \mathrm{C}$ with either a 4-HNE rabbit polyclonal antibody (1:500; Calbiochem, San Diego, CA, U.S.A.), or a antihuman/mouse activated caspase-3 antibody $(1: 1000$; R \& D Systems, Minneapolis, MN, USA). Both antibodies were made up in a diluent consisting of $5 \%$ NGS and $0.1 \%$ Triton-X100 in $0.1 \mathrm{M}$ PBS. The sections were then washed three times in PBS, and incubated for $1 \mathrm{~h}$ at room temperature with a secondary biotinylated anti-rabbit IgG antibody (1:200; Vector Laboratories, Burlingame, CA, U.S.A.). Antibody binding was visualized using the avidin-biotin peroxidase complex (1:200, ABC Vectorstain kit, Vector Laboratories, CA, USA), with metalenhanced diaminobenzidine (Pierce Biotechnology, Rockford, IL, U.S.A.) as the chromogen. Cresyl violet/acid fuchsin staining was carried out on adjacent sections to determine the presence of pyknotic cells.

Double-label immunohistochemistry was carried out sections from two control and two UCO fetal brains by first incubating the sections with either monoclonal anti-GFAP (1/50; F. Hoffmann-La Roche Ltd., Basel, Switzerland), antiCNPase (1/200; Sigma Chemical, St. Louis, MO, U.S.A.) or anti-MAP2 (1/200; Lab Vision-NeoMarkers, Fremont, CA, U.S.A.) to identify astrocytes, oligodendrocyte, and mature neurons, respectively, and then using the rabbit polyclonal antibodies to identify either 4HNE $(1 / 500)$ or activated caspase-3 (1/500). Immunoreactivity (IR) was visualized with Alexa Fluor 594 goat anti-mouse (1/1000; Molecular Probes, Eugene, OR, U.S.A.) and Alexa Fluor 488 goat anti-rabbit (1/1000, Molecular Probes) and assessed with fluorescence microscopy using an Axioplan 2 microscope (400× magnification, Carl Zeiss, Thornwood, NY, U.S.A.). Photographs were taken using an Axiocam-2 camera and the Axiovision 3.1 computer software.

Quantitation. 4-HNE-positive cells were counted under light microscopy (Optipho-2, 400× magnification; Nikon, Tokyo, Japan). Activated caspase-3-positive cells were counted using light microscopy and an MCID image analyzer. Two sections of each brain region per animal were examined for 
4-HNE and activated caspase-3 immunoreactivity. The number of immunopositive cells per region was calculated using the average of four fields of view per section, and the results were averaged across all the animals in each group ( $n=5$ control, $n$ $=6$ occlusion). Pyknotic cells in sections stained with cresyl violet/acid fuchsin, identified as cells showing chromatin condensation and shrunken nuclei and cytoplasm, were counted under light microscopy in four fields of view per section $(n=2$ sections per animal) at $400 \times$ magnification, and the results averaged across all the fetuses in the UCO and control groups. In the double-labeled section, cells that showed immunoreactivity for either MAP2, GFAP or CNPase, and either 4-HNE or caspase- 3 were counted, and the counts averages across at least two sections for each area of interest for each fetus. Cell counts (number of cells per field of view) were rated as either high (26-50), moderate (16-25), low (6-15), or few (0-5).

Statistical evaluation. Significance of changes in fetal blood gases, $\mathrm{pH}$, blood pressure, and heart rate with time and between groups were determined by two-way repeated measures ANOVA followed by a Student-Newman-Keuls posthoc test. Cell counts in the different regions of the UCO and control brains were compared using the unpaired $t$ test followed by a Bonferroni adjustment. Significance was set at $p<0.05$.

\section{RESULTS}

UCO for 10 min produced hypertension and bradycardia and resulted in severe asphyxia and hypoxemia (Fig. 1). There was a biphasic change of arterial pressure - an initial increase from $34.4 \pm 1.9$ to $56.1 \pm 4.1 \mathrm{~mm} \mathrm{Hg}(p<0.05)$ beginning immediately on inflation of the cuffs and reaching a maximum after $4 \mathrm{~min}$ and then decreasing, and a second increase to 49.7 $\pm 2.2 \mathrm{~mm} \mathrm{Hg}(p<0.05)$ on release of the cuffs. Twenty minutes after release of the cuffs, the arterial pressure was not different from control values. Heart rate decreased from $157 \pm$ 11 beats/min immediately on occlusion to reach a nadir or 76 \pm 11 after $4 \min (p<0.05)$; the heart rate increased throughout the remaining time of occlusion to almost reach the preocclusion value by the end of the $10-\mathrm{min}$ period, and then increased further to $210 \pm 13$ beats/min $(p<0.05)$ beginning immediately on release of the cuffs. Heart rate was normal again $1 \mathrm{~h}$ after the occlusion. Arterial $\mathrm{Po}_{2}$ decreased from 25.2 \pm 1.6 to $9.9 \pm 2.4 \mathrm{~mm} \mathrm{Hg}$, and $\mathrm{PCO}_{2}$ increased from $48.7 \pm 1.8$ to $98.6 \pm 4.3 \mathrm{~mm} \mathrm{Hg}$ after $5 \mathrm{~min}$ of occlusion $(p<0.05$; Fig. $1, C$ and $E$ ). There was some recovery from these levels of hypoxia and hypercapnia before the end of the 10-min occlusion, but the decrease of $\mathrm{PO}_{2}$ and increase of $\mathrm{PCO}_{2}$ were still significant compared with the preocclusion baseline values. Similarly, $\mathrm{O}_{2}$ saturation decreased significantly from $73.4 \pm$ $3.5 \%$ to $11.7 \pm 4.8 \%(p<0.05)$ after $5 \mathrm{~min}$ of occlusion, and recovered slightly to $25.2 \pm 9.3 \%$ at $10 \mathrm{~min}$ (Fig. $1 D$ ). Arterial $\mathrm{pH}$ fell from $7.377 \pm 0.015$ to reach its lowest point $7.028 \pm$ $0.061(p<0.05)$ at $10 \mathrm{~min}$ (Fig. 1C). Blood gases and $\mathrm{O}_{2}$ saturation recovered rapidly and were not different from the preocclusion values 20 min after the release of the cuffs, but $\mathrm{pH}$ recovered more slowly and did not reach control values until $1 \mathrm{~h}$ after the occlusion. There were no significant differences in the cardiovascular, blood gas, and acid-base changes produced by the occlusions done on postoperative $\mathrm{d} 3$ and 5. Preocclusion lactate levels increased from $1.1 \pm 0.18$ to $3.8 \pm 1.07$ in occlusion 1 and to $3.5 \pm 0.55$ in occlusion 2 . Lactate levels peaked at $10 \mathrm{~min}$ after UCO and returned to preocclusion levels 90 min after UCO in both occlusion periods.

Lipid peroxidation. 4-HNE immunoreactivity was widely distributed in the fetal brain examined $48 \mathrm{~h}$ after the second UCO (Table 1 and Fig. 2). Cell bodies and fiber tracts were immunopositive for 4-HNE. The staining of cells was particularly prominent in the pyramidal cell layer of CA1 and the pyramidal and polymorphic layer of CA3 regions of the hippocampus (Fig. 2), and in the external granular (layer II), external pyramidal (III), internal granular (IV), and internal pyramidal (V) layers of the parietal cortex (Fig. 2). The 4-HNE immunoreactivity was present in large cells that had the appearance of neuronal cell bodies, and staining was usually present in the nucleus and throughout the cytoplasm. In brains from the control fetuses, 4-HNE immunoreactivity was undetectable (Fig. 2, $A$ and $C$ ). In white matter, many fibers were 4-HNE positive in the UCO brain (Fig. 2, $F$ and $H$ ), whereas 4-HNE immunoreactivity was completely absent in control brains (Fig. 2, $E$ and $G$ ). In white matter, the 4-HNE-positive fibers were intermingled with small 4-HNE-immunopositive cells, which, based on their size and distribution, were likely to be glia (Fig. 2F). In the cerebellum, 4-HNE staining was present in Purkinje cells and cells of the granular and molecular layers, and there was also dense staining of the mossy fiber tracts and, to a lesser extent, the climbing fiber tracts (Fig. 2H). As for other brain areas, 4-HNE positive fibers were not seen in cerebella from control fetuses (Fig. 2G). The number of 4-HNE-positive cells was significantly increased in the hippocampus, cerebellum, parietal cortex, thalamus, putamen, caudate nucleus, and substantia nigra of UCO fetuses compared with and control fetal brains (Table 1).

Double-labeling showed that 4-HNE immunoreactivity was present in cells identified as astrocytes (GFAP-positive), oligodendrocytes (CNPase-positive), or neurons (MAP2-positive) (Fig. 3, $A-F$ ). In control fetuses, few of each cell type (one to five cells per field of view) were immunopositive for 4-HNE in all brain regions examined. After UCO, 4-HNE-positive neurons were present in high numbers (26-50 cells per field) in the hippocampus (Fig. 3, $A$ and $D$ ), and also in parietal cortex, substantia nigra, caudate nucleus, putamen, ventral striatum, thalamus, and in cerebellar Purkinje and granule cell layers (data not shown). Astrocytes and oligodendrocytes were also 4-HNE positive in these regions but at lower levels compared with neurons (astrocytes, 16-25 cells per field; oligodendrocytes, $6-15$ cells per field); examples from the hippocampus of a UCO fetus are shown in Figure 3, B, C, E and $F$.

Apoptosis. Activated caspase- 3 immunoreactivity was present in all brain regions examined in control and UCO fetuses (Table 1 and Fig. 4). UCO resulted in a significant increase in the number of caspase-3-positive cells in the granular and molecular layers of the cerebellar folia, and in the ventro-lateral nucleus of the thalamus, substantia nigra, putamen, and CA1 of the hippocampus. Examples for the hippocampus, cerebral cortex, and cerebellum are shown in Figure 4. Cerebellar Purkinje cells were devoid of caspase-3 immu- 

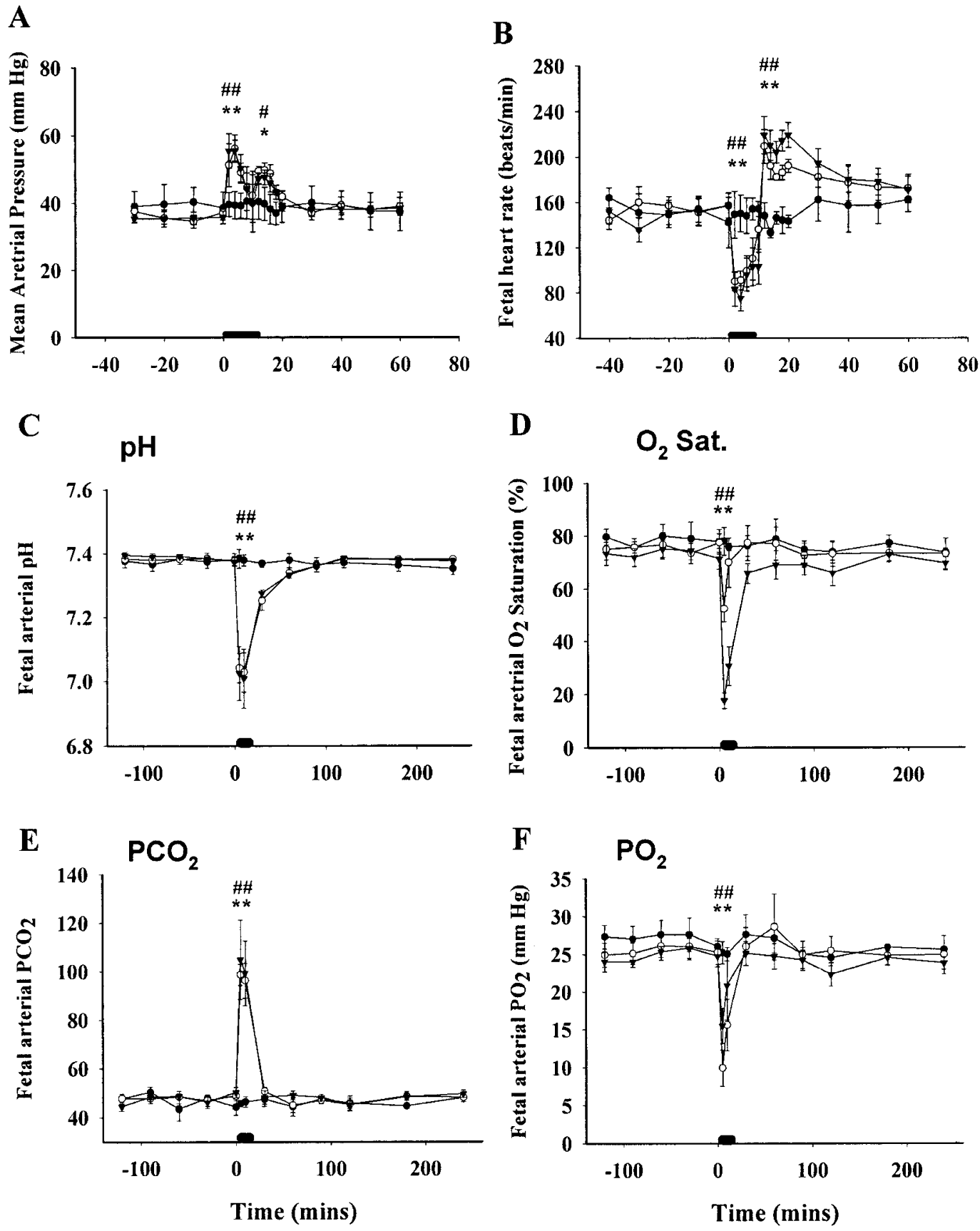

Figure 1. Fetal mean arterial pressure $(A, \mathrm{~mm} \mathrm{Hg})$ and heart rate $(B$, beats $/ \mathrm{min})$ before, during, and after $10 \mathrm{~min}$ occlusion of the umbilical cord. The solid bars indicate the time and duration of the occlusion. In the control experiment, parameters were recorded throughout the day but no occlusion was carried out. Parameters were recorded continuously from $24 \mathrm{~h}$ before to $48 \mathrm{~h}$ after the onset of the cord occlusion, but are shown here over a period of $-40 \mathrm{~min}$ to $80 \mathrm{~min}$ with respect to occlusion. Fetal arterial blood $\mathrm{pH}(C), \mathrm{PCO}_{2}(E, \mathrm{~mm} \mathrm{Hg}), \mathrm{Po}_{2}(F, \mathrm{~mm} \mathrm{Hg})$, and $\mathrm{O}_{2}$ saturation $(D$, \%) before, during, and after $10 \mathrm{~min}$ occlusion of the umbilical cord. The solid bars indicate the time and duration of the occlusion. In control experiment, blood gases were measured throughout the day and at the same time points but no occlusion was carried out. Symbols are as follows: control (solid circle), occlusion 1 (open circle), and occlusion 2 (solid triangle). ${ }^{*} p<0.05$ significant difference between control and occlusion $1 ;{ }^{* *} p<0.01$ significant difference between control and occlusion $1 ; \# p<0.05$ significant difference between control and occlusion $2 ; \# \# p<0.01$ significant difference between control and occlusion 2 .

noreactivity in all UCO and control fetuses. For the majority of the brain regions examined, caspase- 3 immunoreactivity was clearly concentrated in the nucleus of the cell, but cytoplasmic staining was also observed. The incidence of activated caspase3 -positive cells in the different brain regions of the control and UCO fetuses is shown in Table 1. Significant increases occurred in the ventrolateral thalamic nucleus, substantia nigra, putamen, the hippocampal CA1 region, and the granular and molecular layers of the cerebellum. Double-labeling revealed that many caspase-3-positive cells in UCO fetal brains were neurons as shown by medium ( $16-25$ cells per field of view) to high levels (26-50 cells per field of view) of cells immunoreactive to both MAP2 and caspase-3 (Fig. 3, $G$ and $J$ ). The highest levels of such doubly labeled neurons were observed in the putamen and the hippocampus, where in control fetuses only few ( $1-5$ cells per field) to low $(6-15$ cells per field) 
Table 1. Number of cells immunopositive for activated 4-HNE, caspase-3 (cells per $\mathrm{mm}^{2}$ ), and pyknosis in brain regions of control fetuses and in UCO fetuses $48 \mathrm{~h}$ after two episodes of occlusion of the umbilical cord for $10 \mathrm{~min}$.

\begin{tabular}{|c|c|c|c|c|c|c|}
\hline \multirow[b]{2}{*}{ Brain region } & \multicolumn{2}{|c|}{ 4-HNE } & \multicolumn{2}{|c|}{ Caspase-3 } & \multicolumn{2}{|c|}{ Pyknosis } \\
\hline & Control & $\mathrm{UCO}$ & Control & $\mathrm{UCO}$ & Control & $\mathrm{UCO}$ \\
\hline Parietal cortex & $4.9 \pm 1.1$ & $25.4 \pm 3.0^{*}$ & $46.6 \pm 7.1$ & $47.3 \pm 6.6$ & $0.1 \pm 0.0$ & $0.4 \pm 0.0^{* *}$ \\
\hline Stria terminalis & $5.1 \pm 1.4$ & $13.2 \pm 1.5$ & $48.0 \pm 9.3$ & $45.3 \pm 4.7$ & 0 & 0 \\
\hline \multicolumn{7}{|l|}{ Thalamus } \\
\hline Ventrolateral th nu & $5.1 \pm 0.4$ & $9.1 \pm 0.9 * *$ & $34.2 \pm 6.1$ & $65.1 \pm 2.5^{*}$ & 0 & 0 \\
\hline Reticular th nu & $5.4 \pm 2.4$ & $18.0 \pm 1.8^{*}$ & $32.0 \pm 8.3$ & $37.1 \pm 7.2$ & 0 & 0 \\
\hline Paraventricular th $\mathrm{nu}$ & $2.7 \pm 0.9$ & $7.3 \pm 2.0$ & $63.1 \pm 1.2$ & $60.6 \pm 1.8$ & 0 & 0 \\
\hline Posteromedial th nu & $1.8 \pm 0.5$ & $9.1 \pm 0.9^{* *}$ & 0 & 0 & 0 & 0 \\
\hline Substantia nigra & $1.5 \pm 0.9$ & $9.7 \pm 1.6^{*}$ & $42.3 \pm 3.6$ & $92.0 \pm 3.7 * *$ & 0 & 0 \\
\hline \multicolumn{7}{|l|}{ Striatum } \\
\hline Ventral striatum & $8.4 \pm 2.0$ & $30.6 \pm 4.1 *$ & $30.4 \pm 4.8$ & $33.4 \pm 2.3$ & 0 & 0 \\
\hline Putamen & $6.8 \pm 0.8$ & $26.1 \pm 2.5^{* *}$ & $24.2 \pm 2.3$ & $39.4 \pm 0.8^{*}$ & $0.13 \pm 0.0$ & $0.5 \pm 0.1 * *$ \\
\hline Caudate nu & $3.7 \pm 1.8$ & $28.6 \pm 2.5^{*}$ & $79.1 \pm 13.4$ & $79.3 \pm 17.6$ & $0.1 \pm 0.0$ & $0.5 \pm 0.0^{* *}$ \\
\hline \multicolumn{7}{|l|}{ Hippoccampus } \\
\hline CA1 & $3.7 \pm 1.4$ & $15.0 \pm 2.0^{*}$ & $58.5 \pm 12.6$ & $111.3 \pm 18.5^{* *}$ & $0.2 \pm 0.1$ & $0.7 \pm 0.1^{*}$ \\
\hline CA3 & $4.2 \pm 2.0$ & $12.7 \pm 1.6^{*}$ & $114.1 \pm 9.8$ & $123.2 \pm 10.1$ & $0.2 \pm 0.1$ & $1.8 \pm 0.1^{* *}$ \\
\hline Molecular layer & $2.6 \pm 1.0$ & $12.0 \pm 3.3^{*}$ & $90.8 \pm 20.1$ & $115.9 \pm 24.2^{*}$ & $0.1 \pm 0.0$ & $0.4 \pm 0.2$ \\
\hline Dentate gyrus & $3.3 \pm 1.1$ & $18.6 \pm 3.7 *$ & $49.0 \pm 4.5$ & $81.1 \pm 20.1 *$ & $0.1 \pm 0.0$ & $0.2 \pm 0.0$ \\
\hline \multicolumn{7}{|l|}{ Cerebellum } \\
\hline Purkinje cell layer & $0.8 \pm 0.0$ & $6.1 \pm 1.6^{*}$ & 0 & 0 & $0.4 \pm 0.0$ & $2.0 \pm 0.0^{* *}$ \\
\hline Molecular layer & $0.8 \pm 0.6$ & $12.3 \pm 1.3^{*}$ & $62.3 \pm 12.2$ & $109.5 \pm 11.1^{*}$ & $1.3 \pm 0.0$ & $2.0 \pm 0.1$ \\
\hline Granular layer & $0.2 \pm 0.0$ & $5.6 \pm 0.9^{* *}$ & $57.3 \pm 10.8$ & $93.5 \pm 10.7 *$ & $2.5 \pm 0.4$ & $3.9 \pm 0.8$ \\
\hline
\end{tabular}

Values expressed as mean \pm SEM.

$* p<0.05 ; * *<<0.01$ (unpaired $t$ test).

numbers of such cells were observed. Caspase-3 immunoreactivity was also present in astrocytes (GFAP-positive cells) after $\mathrm{UCO}$, with the greatest numbers in substantia nigra, dentate gyrus, hippocampal molecular layer, cerebellar granule layer, and subcallosal bundle (Fig. 3, $I$ and $L$ ). Oligodendrocytes (CNPase-positive cells) were also caspase-3 positive, with the highest numbers (16-25 cells per field of view) present in the ventrolateral thalamic nucleus, hippocampal molecular layer, and the subcallosal bundle (Fig. 3, $I$ and $L$ ). In control fetuses, few oligodendrocytes were immunopositive for caspase-3 (less than five cells per field of view).

Pyknosis. After UCO cells with pyknotic features (i.e. shrunken cells with condensed, aggregated dark purple chromatin and bright pink, acidotic cytoplasmic staining) were evident in the hippocampus (Fig. $5 C, 5 E$ ), cortex and the cerebellum (Fig. $5 D, 5 F$ ). Few of these cells were observed in control brains $(5 A, 5 B)$. A significant increase in the number of pyknotic cells after UCO was observed in CA1 and CA3 of the hippocampus, layers II and III of the parietal cortex, the putamen of the striatum, and in the Purkinje cell layer of the cerebellum (Table 1).

\section{DISCUSSION}

The present study was designed to determine the distribution 4-HNE (a marker of lipid peroxidation) and caspase-3 (a marker of apoptotic cell death) in the fetal sheep brain after asphyxia produced by UCO. In fetuses subjected to two such brief insults, 4-HNE immunoreactivity was significantly increased in the parietal cortex, striatum, substantia nigra, thalamus, hippocampus, and cerebellum. Based on cell morphology and double labeling with markers for neurons, astrocytes, and oligodendrocytes, this 4-HNE immunoreactivity was found in all cell types, but predominantly in neurons. However, we were unable identify oligodendroblasts in this study due to the lack of a suitable marker for immature oligodendrocytes for sheep CNS and therefore have not determined whether these cells undergo lipid peroxidation after UCO.

Nuclear and cytoplasmic 4-HNE staining in Purkinje cells has been observed previously in the human fetal cerebellum and attributed to a unique aspect of metabolism of the developing brain (18). The absence of any significant 4-HNE immunoreactivity in control fetal sheep brains in the present study suggests that 4-HNE is not normally produced in appreciable amounts by the developing brain, and, thus, oxidative (or other) stresses may have been present during the collection of this human fetal material. A recent study in gerbils using transient ischemia showed that 4-HNE immunoreactivity appeared first in the nuclei of hippocampal neurons at approximately $2 \mathrm{~h}$ of reperfusion, and subsequently in the cytoplasm, where staining persisted for up to $3 \mathrm{~d}$ (15). The early accumulation of 4-HNE in the nucleus is thought to initiate expression of death-effector genes, which then proceeds to programmed (apoptotic) cell death. The gradual accumulation of lipid peroxidation products in cytoplasmic organelles eventually leads to mitochondrial dysfunction (15). In the present study, 4-HNE immunoreactivity was observed in both the nucleus and cytoplasm in many brain regions suggesting that the conditions that lead to lipid peroxidation may be present for some time after the asphyxial events associated with the cord occlusion are resolved. In the gerbil brain, 4-HNE immunoreactivity was found in reactive astrocytes $1 \mathrm{wk}$ after a transient ischemic insult (15).

The importance of perinatal white matter damage after hypoxia-asphyxia has been highlighted many times $(1,19-21)$. 

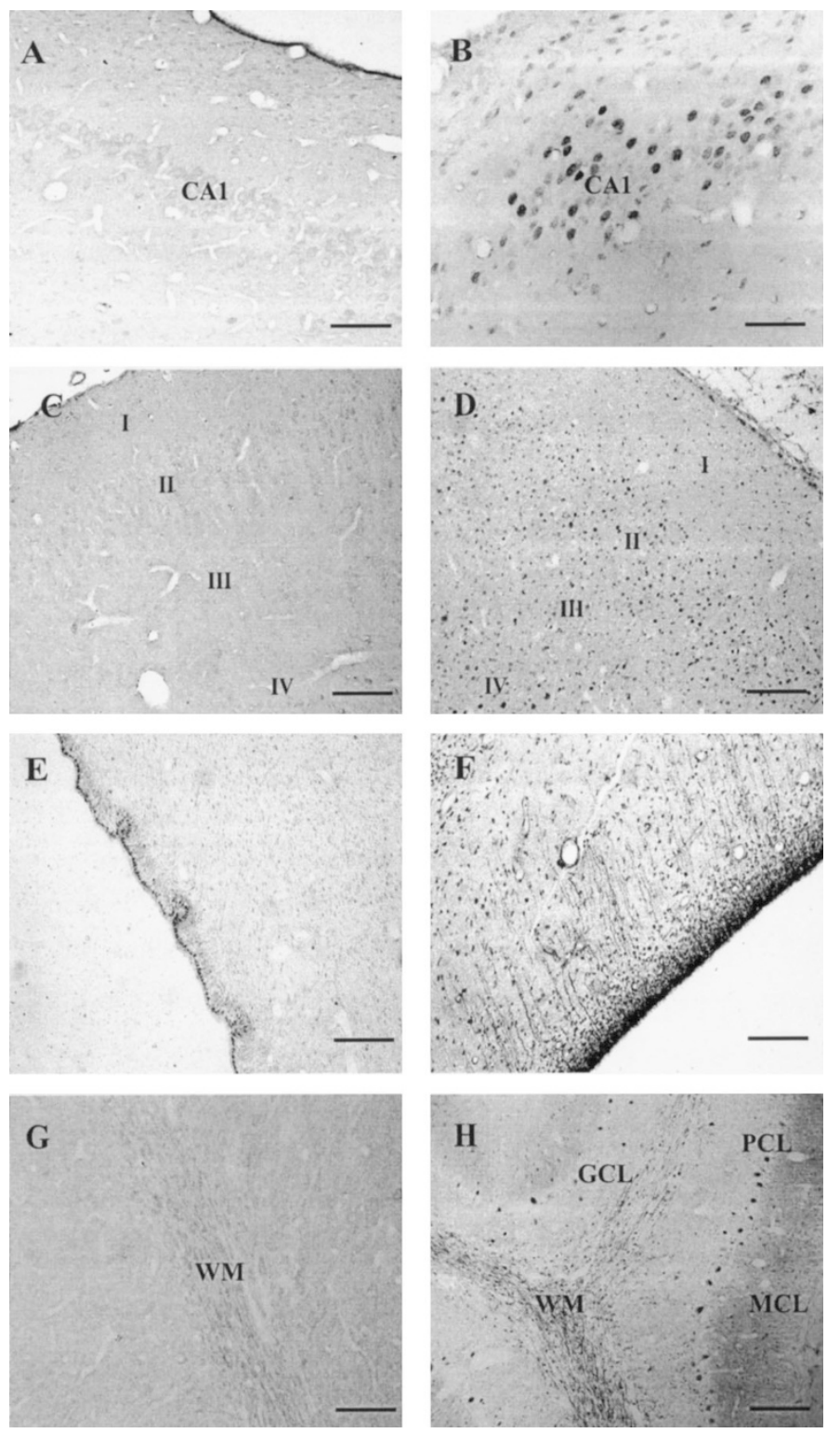

Figure 2. Photomicrographs showing 4-HNE immunopositive cells in control (left panels) and UCO fetuses (right panels) $48 \mathrm{~h}$ after complete occlusion of the umbilical cord for $10 \mathrm{~min}$ on two occasions. $(A, B)$ CA1 region of the hippocampus; $(C, D)$ parietal cortex, showing layers I, II, III, and IV; $(E, F)$ subcallosal bundle; $(G, H)$ cerebellum, showing control brain devoid of 4-HNE but distinct straining in Purkinje cells and mossy and climbing fibers of white matter in UCO fetuses. MCL, molecular cell layer; GCL, granule cell layer; PCL, Purkinje cell layer; WM, white matter. Scale bar $=20 \mu \mathrm{m}(A, B, G, H)$, and $50 \mu \mathrm{m}(C-F)$.

In the present study, UCO increased 4-HNE staining in fiber tracts of the fetal sheep brain, such as the subcallosal bundle, external medullary lamina, fiber tracts of the reticular thalamic nucleus, and in cerebellar white matter. 4-HNE is known to disrupt microtubules, an essential component of fast axonal transport, by binding to microtubular associated protein (22, 23 ), and causes conformational changes in the structure of $\alpha$-tubulin, a major component of microtubules (24). 4-HNE is cytotoxic to oligodendrocytes in culture, and when injected intracerebrally causes axonal damage (14). These observations suggest a possible role for lipid peroxidation in the develop-
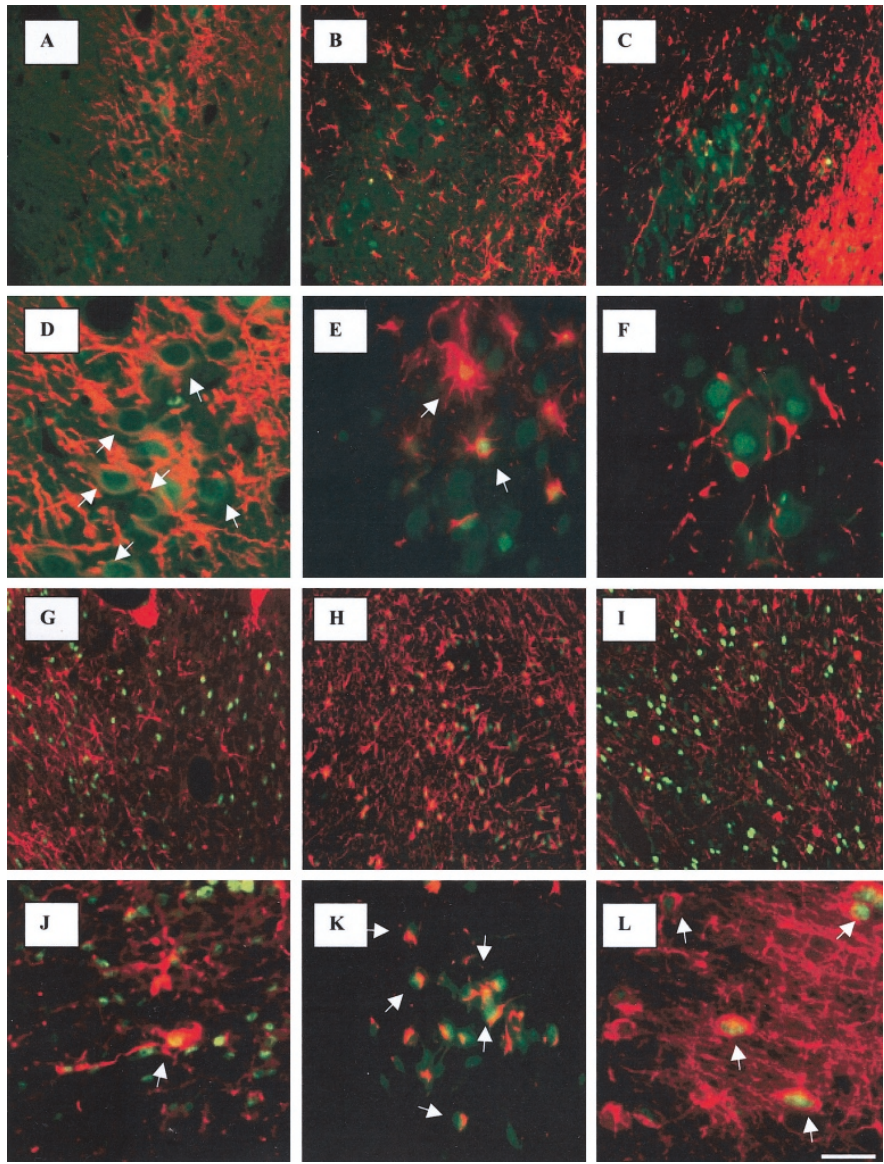

Figure 3. Photomicrographs of the showing dual filter image (double label immunostaining) of MAP2-labeled neurons $(A)$, GFAP-labeled astrocytes $(B)$ and CNPase-labeled oligodendrocytes $(C)$ in the CA1 region of the hippocampus from a UCO fetal brain immunoreactive to 4-HNE. MAP2, GFAP, and CNPase were visualized with Alexa Fluor 594 (red) and 4-HNE was visualized with Alexa Fluor 488 (green). Panels $D, E, F$ are a higher magnification of $A$, $B$, and $C$, respectively. Panels $G, H, I$ are photomicrographs of the subcallosal bundle from a UCO fetal brain showing double-immunostaining for activated caspase-3 (green) and either MAP2 $(G)$, GFAP $(H)$, or CNPase $(I)$ immunoreactive cells. Panels $J, K, L$ show a higher magnification of $G, H$, and $I$, respectively. Arrows in panels $D, E, J, K$, and $L$ point to cells immunopositive for either 4-HNE or caspase-3 and the specific cell marker. Scale bar $=25 \mu \mathrm{m}$ $(A-C, G-I)$ and $50 \mu \mathrm{m}(D-F, J-L)$.

ment of white matter injury. Hypoxic/ischemic insult may preferentially injure oligodendroblasts (oligodendrocytes' precursor cells) leading to long-term myelination deficits in the corpus callosum, cerebellum, and hippocampus $(19-21,25)$. In the present study, intense 4-HNE staining was found in small cells in white matter that had the appearance of glia (oligodendrocytes and astrocytes). Double labeling with specific markers for neurons (anti-MAP2), astrocytes (anti-GFAP), and oligodendrocytes (anti-CNPase) confirmed that 4-HNE immunoreactivity was present in a large number of neurons and astrocytes in all brain regions examined, but relatively small numbers of oligodendrocytes were immunopositive for 4-HNE in the UCO fetal brain. Thus, after this brief asphyctic insult lipid peroxidation appears to occur preferentially in neurons and astrocytes, consistent to some extent with the previous study of human fetal brain that showed 4-HNE immunoreactivity only in astrocytes and neurons (18). 

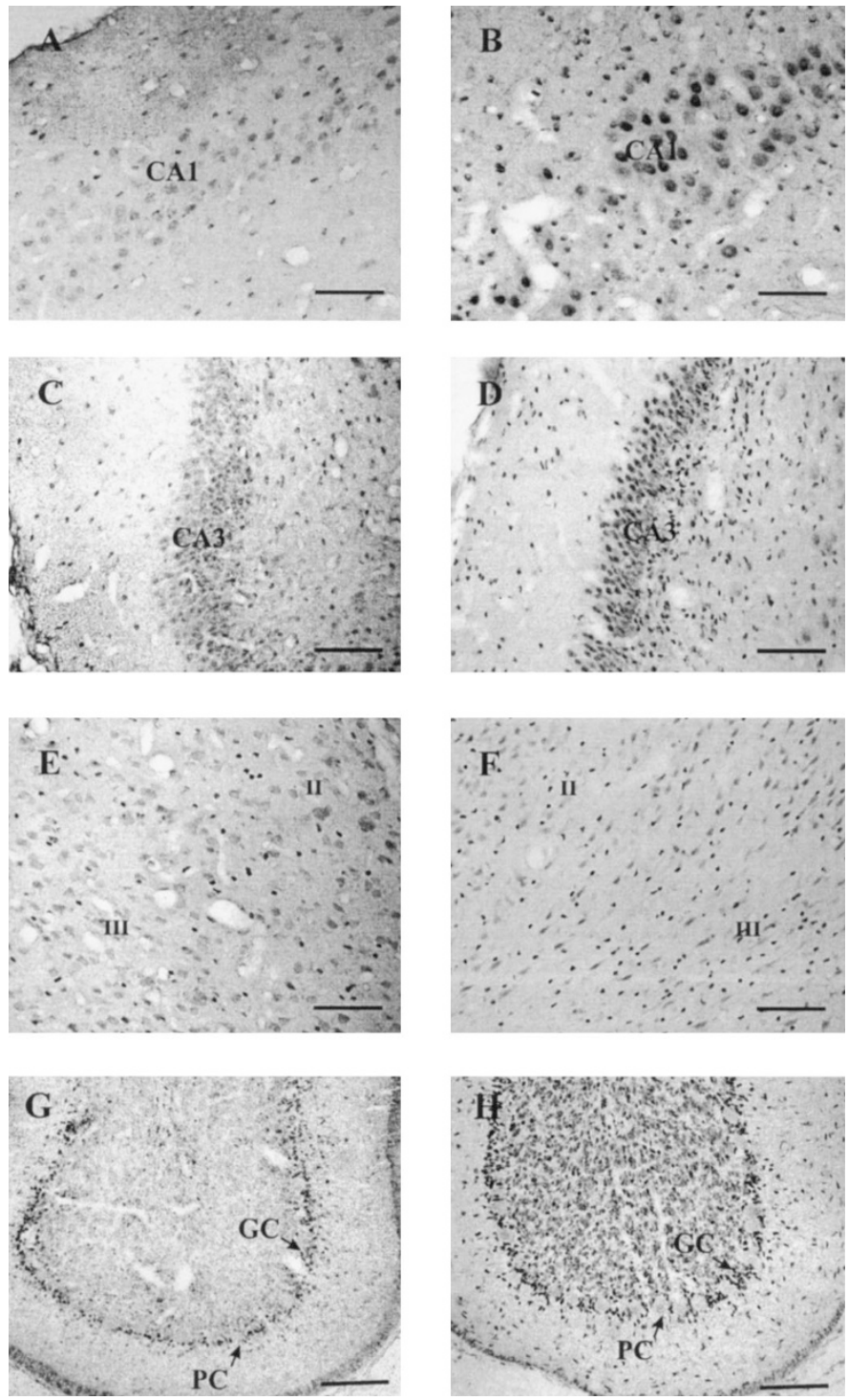

Figure 4. Photomicrographs showing activated caspase- 3 immunopositive cells in control (left panels) and UCO fetuses (right panels) $48 \mathrm{~h}$ after complete occlusion of the umbilical cord for $10 \mathrm{~min}$ on two occasions. Cells in the CA1 $(A, B)$ and $\mathrm{CA} 3(B, D)$ regions of the hippocampus; $(E, F)$ parietal cortex, showing layers II and III; $(G, H)$ cerebellum. PC, Purkinje cells; GC, granular cells. Scale bar $=20 \mu \mathrm{m}(A, B, E, F) ; 50 \mu \mathrm{m}(C, D) ; 70 \mu \mathrm{m}(G, H)$.

Antibody to activated caspase-3 was chosen because it specifically recognizes the "cleaved" or activated form of the protein. Caspase-3 immunoreactivity was present in a number of brain regions in the control fetuses. A similar distribution of activated caspase- 3 was observed in brains collected from fetal sheep that had not undergone surgery (data not shown), ruling out the possibility that the presence of this protein was an artifact due to the surgery and seven postoperative survivals. Apoptotic neuronal death is a normal developmental event in the CNS $(26,27)$. The presence of considerable numbers of caspase- 3 positive cells was observed in various brain regions of control fetuses and suggests that the brain is still undergoing significant modification through programmed cell death at 0.8 of gestation in sheep.

After the brief asphyxia associated with UCO, the number of caspase-3-immunopositive cells was increased significantly in
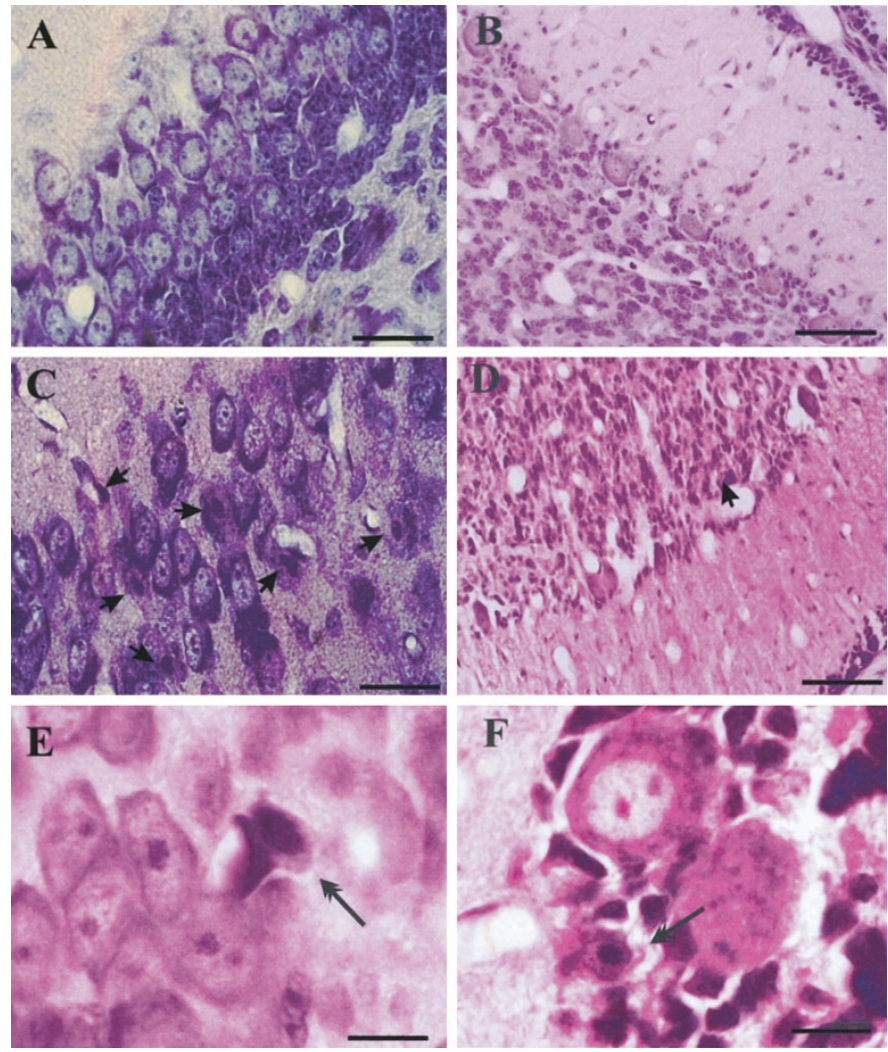

Figure 5. Photomicrographs showing pyknotic cells in the CA1 of the hippocampus from a control $(A)$ and a cord occluded fetus $(C)$. (E) A magnified dead cell in CA1 showing chromatin condensation, shrunken nucleus, and reduced cytoplasm. ( $B$ and $D$ ) Pyknotic cells in the cerebellum of a control and a cord occluded fetus respectively. $(F)$ A magnified dead cell in the Purkinje cell layer of the cerebellum. The arrows in $C-F$ point at pyknotic cells.

regions associated with primary sensory and forebrain motor systems. Significant increases in the number of caspase-3positive cells were observed in the ventrolateral thalamic nucleus, substantia nigra, putamen, the CA1 of the hippocampus, and the granule and molecular cell layers of the cerebellum. Caspase- 3 was present in neurons in gray matter, and it also labeled small cells in white matter, which double-label immunohistochemistry confirmed as oligodendrocytes and astrocytes. In the hippocampus and striatum, the increased caspase-3 immunoreactivity occurred primarily in neurons, whereas in other brain regions (ventrolateral thalamic nucleus, substantia nigra, and the molecular and granule cell layers of the cerebellum) significant numbers of astrocytes and oligodendrocytes were also caspase-3 positive. The caspase-3 staining was present in both nuclei and the cytoplasm in the UCO animals. It has been proposed that cytoplasmic staining with immunonegative nuclei is seen at early stages after induction of apoptosis, whereas whole-cell labeling occurs as the cell progresses toward death (28). The present results suggest that although many cells in the UCO fetal brain had progressed toward end-stage apoptosis at the time of postmortem $(48 \mathrm{~h}$ after the second of the two occlusions), other cells had entered into the apoptotic cascade more recently.

Recent in vitro (29) and in vivo (30) experiments suggest that apoptosis plays a pivotal role in the death of oligodendro- 
cytes induced by hypoxia/ischemia. Although oligodendrocytes did not accumulate 4-HNE to the same extent as neurons and astrocytes, a significant number of them were induced to enter apoptosis, which might limit further myelination of the brain. Loss of astrocytes is also likely to be important in this regard as they are important for white matter architecture and decreased GFAP expression is associated with CNS dysmyelination (31).

The increased cell death after two bouts of UCO was confirmed by the cresyl violet/acid fuchsin staining, which revealed a significant increase in the number of pyknotic cells in the hippocampus (CA1 and CA3), cortex, caudate nucleus, and putamen. These findings are consistent with previous studies showing that hypoxic insult leads to damage and selective neuronal loss in the hippocampal CA1 (32-34) and striatum $(12,32,35)$ in late gestation fetal sheep brain. Pyknotic cells were also found in the cerebellar Purkinje cell layer, where 4-HNE but not caspase-3 immunoreactivity was prominent. This suggests that Purkinje cells either succumb more quickly to caspase-mediated cell death compared with other regions of the brain, or that they are vulnerable to damage that leads directly to cell death and necrosis.

Although distinguishing between apoptosis and necrosis was not a primary objective of this study, it is likely that both processes overlap in time and region after hypoxic/ischemic or asphyctic events in the developing brain $(16,35)$. Early cell death may be necrotic whereas delayed neuronal death is an apoptosis-driven event $(16,17)$. The duration and severity of asphyxia, particularly with respect to effects on local tissue perfusion, oxygenation, accumulation of ROS, and products of lipid peroxidation, are therefore likely to be critical in determining the balance of necrotic and apoptotic effects in the brain. Lipid peroxidation may be a significant early response to asphyxia that leads to cell death and brain damage in the fetus and newborn.

Acknowledgment. The authors thank Alex Satragno for his invaluable surgical expertise.

\section{REFERENCES}

1. Volpe JJ 2001 Perinatal brain injury: from pathogenesis to neuroprotection. Ment Retard Dev Disabil Res Rev 7:56-64

2. Berger R, Garnier Y 1999 Pathophysiology of perinatal brain damage. Brain Res Brain Res Rev 30:107-134

3. Dammann O, Durum S, Leviton A 2001 Do white cells matter in white matter damage? Trends Neurosci 24:320-324

4. Phillis J 1994 A radical view of cerebral ischaemic injury. Prog Neurobiol 42:441448

5. Chandra J, Samali A, Orrenius S 2000 Triggering and modulation of apoptosis by oxidative stress. Free Radic Biol Med 29:323-333

6. Esterbauer H, Schaur RJ, Zollner H 1991 Chemistry and biochemistry of 4-hydroxynonenal, malonaldehyde and related aldehydes. Free Radic Biol Med 11:81-128

7. Maulik D, Zanelli S, Numagami Y, Ohnishi ST, Mishra OP, Delivori a-Papadopoulos M 1999 Oxygen free radical generation during in-utero hypoxia in the fetal guinea pig brain: the effect of maturity and of magnesium sulfate administration. Brain Res $817: 117-122$

8. Bagenholm R, Nilsson UA, Gotborg CW, Kjellmer I 1998 Free radicals are forme in the brain of fetal sheep during reperfusion after cerebral ischemia. Pediatr Res 43:271-275
9. Masaoka N, Hayakawa Y, Ohgame S, Sakata H, Satoh K, Takahashi H 1998 Changes in purine metabolism and production of oxygen free radicals by intermittent partial umbilical cord occlusion in chronically instrumented fetal lambs. J Obstet Gynaecol Res 124:63-71

10. Ikeda T, Choi BH, Yee S, Murata Y, Quilligan EJ 1999 Oxidative stress, brain white matter damage and intrauterine asphyxia in fetal lambs. Int J Dev Neurosci 17:1-14

11. Bennet L, Rossenrode S, Gunning MI, Gluckman PD, Gunn AJ 1999 The cardiovascular and cerebrovascular responses of the immature fetal sheep to acute umbilical cord occlusion. J Physiol 517:247-257

12. Ikeda T, Murata Y, Quilligan EJ, Choi BH, Parer JT, Doi S, Park SD 1998 Physiologic and histologic changes in near-term fetal lambs exposed to asphyxia by umbilical cord occlusion. Am J Obstet Gynecol 178:24-32

13. Kruman I, Bruce-Keller AJ, Bredesen D, Waeg G, Mattson MP 1997 Evidence that 4-hydroxynonenal mediates oxidative stress-induced neuronal apoptosis. J Neurosci 17:5089-5100

14. McCracken E, Valeriani V, Simpson C, Jover T, McCulloch J, Dewar D 2000 The lipid peroxidation by-product 4-hydroxynonenal is toxic to axons and oligodendrocytes. J Cereb Blood Flow Metab 20:1529-1536

15. Urabe T, Yamasaki Y, Hattori N, Yoshikawa M, Uchida K, Mizuno Y 2000 Accumulation of 4-hydroxynonenal-modified proteins in hippocampal CA1 pyramidal neurons precedes delayed neuronal damage in the gerbil brain. Neuroscience 100:241-250

16. Martin LJ, Brambrink AM, Price AC, Kaiser A, Agnew DM, Ichord RN, Traystman RJ 2000 Neuronal death in the newborn striatum after hypoxia-ischemia is necrosis and evolves with oxidative stress. Neurobiol Dis 7:169-191

17. Northington FJ, Ferriero DM, Graham EM, Traystman RJ, Martin LJ 2001 Early neurogeneration after hypoxia-ischemia in neonatal rat is necrosis while delayed neuronal death is apoptosis. Neurobiol Dis 8:207-219

18. Yamamoto T, Shibata N, Muramatsu F, Sakayori N, Kobayashi M 2002 Oxidative stress in the human fetal brain: an immunohistochemical study. Pediatr Neurol 26:116-122

19. Jelinski SE, Yager JY, Juurlink BH 1999 Preferential injury of oligodendroblasts by a short hypoxic-ischemic insult. Brain Res 815:150-153

20. Kohlhauser C, Mosgoller W, Hoger H, Lubec B 2000 Myelination deficits in brain of rats following perinatal asphyxia. Life Sci 67:2355-2368

21. Skoff RP, Bessert DA, Barks JD, Song D, Cerghet M, Silverstein FS 2001 Hypoxicischemic injury results in acute disruption of myelin gene expression and death of oligodendroglial precursors in neonatal mice. Int J Dev Neurosci 19:197-208

22. Mattson MP, Fu W, Waeg G, Uchida K 1997 4-Hydroxynonenal, a product of lipid peroxidation, inhibits dephosphorylation of the microtubule-associated protein tau Neuroreport 8:2275-2281

23. Neely MD, Sidell KR, Graham DG, Montine TJ 1999 The lipid peroxidation product 4-hydroxynonenal inhibits neurite outgrowth, disrupts neuronal microtubules, and modifies cellular tubulin. J Neurochem 72:2323-2333

24. Subramaniam R, Roediger F, Jordan B, Mattson MP, Keller JN, Waeg G, Butterfield DA 1997 The lipid peroxidation product, 4-hydroxy-2-trans-nonenal, alters the conformation of cortical synaptosomal membrane proteins. J Neurochem 69:11611169

25. Back SA, Luo NL, Borenstein NS, Levine JM, Volpe JJ, Kinney HC 2001 Late oligodendrocyte progenitors coincide with the developmental window of vulnerability for human perinatal white matter injury. J Neurosci 21:1302-1312

26. Oppenheim RW 1991 Cell death during development of the nervous system. Annu Rev Neurosci 14:453-501

27. Mooney SM, Miller MW 2000 Expression of bcl-2, bax, and caspase-3 in the brain of the developing rat. Brain Res Dev Brain Res 123:103-117

28. Tenneti L, Lipton SA 2000 Involvement of activated caspase-3-like proteases in $\mathrm{N}$-methyl-D-aspartate-induced apoptosis in cerebrocortical neurons. J Neurochem $74: 134-142$

29. Shibata M, Hishara S, Hara H, Yamawaki T, Fukuuchi Y, Yuan J, Okano H, Miura M 2000 Caspases determine the vulnerability of oligodendrocytes in ischemic brain. J Clin Invest 106:643-653

30. Han BH, D'Costa A, Back SA, Parsadanian M, Patel S, Shah AR, Gidday JM, Srinivasan A, Deshmukh M, Holtzman DM 2000 BDNF blocks caspase-3 activation in neonatal hypoxia-ischemia. Neurobiol Dis 7:38-53

31. Liedtke W, Edelmann W, Bieri PL, Chiu FC, Cowan NJ, Kucherlapati R, Raine CS 1996 GFAP is necessary for the integrity of CNS white matter architecture and long-term maintenance of myelination. Neuron 17:607-615

32. Gunn AJ, Parer JT, Mallard EC, Williams CE, Gluckman PD 1992 Cerebral histological and electrophysiological changes after asphyxia in fetal sheep. Pediatr Res 31:486-491

33. Mallard EC, Gunn AJ, Williams CE, Johnson BM, Gluckman PD 1992 Transient umbilical cord occlusion causes hippocampal damage in fetal sheep. Am J Obstet Gynecol 167:1423-1430

34. Mallard EC, Williams CE, Johnson BM, Gluckman PD 1994 Increased vulnerability to neuronal damage after umbilical cord occlusion in fetal sheep with advancing gestation. Am J Obstet Gynecol 170:206-214

35. Mallard EC, Williams CE, Johnson BM, Gunning MI, Davis S, Gluckman PD 1995 Repeated episodes of umbilical cord occlusion in fetal sheep lead to preferentia damage of the striatum and sensitize the heart to further insults. Pediatr Res 37:707-713 